\title{
Theoretical Analysis of Single-blowing Operation in Hot-blast Stoves*
}

\author{
By Masayuki HORIO** and Iwao MUCHI**
}

\section{Synopsis}

Two methods to solve the two point boundary value problem of hot-blast stoves for the case whereat the mass flow rate of air during the cooling period changes are presented on the basis of the approximate analysis concerning the balanced cycle operations.

Each solution consists of two parts: the first is a necessary condition for a balanced cycle and the second is a set of equations which make it possible to determine the average flow rate of air during the cooling period and the longitudinal distribution of temperature in the checker. Accuracy and validity of these approximate solutions are shown by the numerical examples.

Simple nomograms for the stove calculations are presented on the basis of the approximate solutions and by the use of these monograms the characteristics of stove operations are discussed. Furthermore, a numerical example to determine the heating surface area of the checker is given.

\section{Introduction}

Since the heat-exchange process of hot-blast stoves is a cyclic process, the analysis of hot-blast stoves is rather difficult in comparison with the analysis of recuperators.

Analytical solutions for the problems of blast stoves or of regenerators have been presented by many investigators. ${ }^{1-6)}$ In the derivations of these analytical solutions, it is necessary to obtain a solution of the partial differential equations for arbitrary initial distribution of brick temperature and to get an integral equation by substituting the solution into the equation representing the condition for cyclic equilibrium, i.e., so called reversal condition. Then the initial distributions of brick temperature in the heating period and of that in the cooling period can be given as the solution of the integral equation. Since the analytical solutions are complicated in most cases, the calculation procedure based on them may be too laborious to apply them to the practical problems.

In the 1960's the simulation methods ${ }^{7,8)}$ of blast stoves with the aid of an electronic computer were developed. The difficulties mentioned above in the analytical treatment can be avoided by the numerical simulation, but there arises a new problem, e.g., how to reduce the time for computation.

To solve the problems more easily, some approximate methods have been presented. The overall heat transfer coefficient introduced by Schack ${ }^{9)}$ makes it possible to have an analogy with a recuperator, if the actual flow rate of air is given. However, to determine the flow rate of air and to obtain a complete solution of the regenerator problem, it is necessary to take account of the end effect of the checkerwork. Recently Hausen ${ }^{10)}$ has presented an approximate method to treat the entrance regions of the checkerwork, but the entire approximate solution for the two point boundary value problem has not been presented.

In this paper, the single-blowing operations in hotblast stoves are treated by both numerical computation and approximate analysis. In the approximate analysis we have focused on a "balanced cycle operation " wherein the cumulative amount of heat capacity of gas flowing through the checkerwork during the heating period is equal to that in the cooling period and also the thermal efficiencies in both periods are equal each other. In this operation the longitudinal distributions of temperature become linear in the checkerwork except the entrance regions. Many commercial stoves are considered to be operated under the conditions near the balanced cycle, since the linear temperature profiles have been obtained in the empirical measurements. ${ }^{11-13)}$

Two kinds of simple solutions, A and B described later, for the two point boundary value problem in the cyclic operation of regenerators are derived analytically in this paper. These solutions include the criterion equation to estimate whether or not a balanced cycle operation is attained under a given operating conditions. Since the operations under the balanced cycle are near optimal as described by Green, ${ }^{14}$ and are desirable in the practical operation, the solutions for the balanced cycle given in the following may be applicable for the practical problems.

\section{Mathematical Model}

\section{Fundamental Equations}

The fundamental equations are written on the basis of the following assumptions.

(1) For the sake of the stationary operation of blast furnace the blast volume and the blast temperature are maintained at constant values by regulating the flow rate of by-passing air.

(2) The stoves are operated under a cyclic equilibrium, and so it is sufficient to analyze only one cycle.

(3) The flow rate of hot gas (i.e., product of combustion) is constant.

(4) The inlet temperatures of air and of hot gas are constant.

(5) Overall heat transfer coefficient is considered to be constant during each period.

(6) Specific heats of gas and solid are constant, respectively.

(7) The heat accumulation of gas contained in

* Originally published in Tetsu-to-Hagané, 58 (1972), 1355 and 59 (1973), 702, in Japanese. English version received October $19,1973$.

** Department of Iron and Steel Engineering, Nagoya University, Chikusa-ku, Nagoya 464. 
the flues is negligible.

(8) Heat loss from the wall is negligible.

(9) Gas flow is considered as a piston flow.

(10) Longitudinal heat conduction in the checkerwork can be neglected.

Most of the assumptions described above have been used by the other investigators. ${ }^{1-8,10-12,14-16)}$

The thermal behavior of hot-blast stoves can be described by the differential Eqs. (1) and (2).

$$
\begin{aligned}
\partial t / \partial \tau & =H \Lambda(T-t) \ldots \\
\partial T / \partial \zeta & =(\Lambda / v)(t-T)
\end{aligned}
$$

where, $t$ and $\mathcal{T}$ designate the brick temperature and the gas temperature, $\tau$ is the dimensionless time and $\zeta$ is the dimensionless height from the lower end of the checkerwork. These variables are defined by Eq. (3).

$$
\left.\begin{array}{c}
\tau=\theta \mid \Theta_{c}, \quad \zeta=z / L, \\
t(\tau, \zeta)=\left(t^{\prime}-T_{a}^{\prime}\right) /\left(T_{g}^{\prime}-T_{a}^{\prime}\right), \\
T(\tau, \zeta)=\left(T^{\prime}-T_{a}^{\prime}\right) /\left(T_{g}^{\prime}-T_{a}^{\prime}\right)
\end{array}\right\}
$$

The boundary conditions are specified by Eqs. (4) to $(6)$.

$$
\begin{aligned}
T(\tau, 0) & \equiv T_{c 0}=0 \quad(\mathrm{CP}) . \\
T(\tau, 1) & \equiv T_{h 1}=1 \quad(\mathrm{HP}) . \\
t(0, \zeta) & =t(1+\gamma, \zeta)
\end{aligned}
$$

Equation (6) represents the condition for a cyclic equilibrium.

The dimensionless factors $H$ and $A$ appeared in Eqs. (1) and (2) are defined by Eq. (7).

$$
\left.\begin{array}{rlrl}
H_{c} & =W_{b} c_{a} \Theta_{c} / M c_{s}, & H_{h} & =W_{g} c_{g} \Theta_{c} / M c_{s}, \\
\Lambda_{c} & =h_{c}^{*} A / W_{b} c_{a}, & \Lambda_{h} & =h_{h}^{*} A / W_{g} c_{g}
\end{array}\right\}
$$

Now, we define $\gamma$ as given by Eq. (8), and then the duration of full cycle becomes $1+\gamma$.

$$
\gamma=\Theta_{h} \mid \Theta_{c}=\mathcal{N}-1-\Delta \tau_{c h}
$$

where, $\mathcal{N}$ is the number of stoves in a system of hotblast stoves and $\Delta \tau_{c h}$ is the time for the change over. The origin of $\tau$ is placed at the beginning of cooling period (cf., Fig. 1).

On the basis of the assumptions (1) and (3) the dimensionless flow rate of gas, $v$, is determined by Eqs. (9a) or $(9 b)$.

$$
v=\left\{\begin{array}{l}
\mathcal{T}_{b} / \mathcal{T}_{c 1}(\tau)=u(\tau) \\
-1
\end{array}\right.
$$

where, $T_{b}$ is the dimensionless blast temperature.

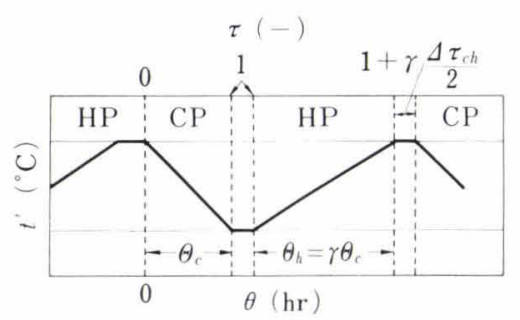

Fig. 1. Illustration of the cyclic behavior of brick temperature
When $v$ is positive, the gas flows up from the lower entrance of the checkerwork, and when $v$ is negative, the gas flows down.

\section{Thermal Efficiency}

From the overall heat balance concerning one cycle, Eqs. (10) and (11) representing the thermal efficiency of each period can be obtained.

$$
\begin{aligned}
& \eta_{c}=T_{b} / \bar{u} \fallingdotseq T_{c 1} \ldots \\
& r_{h}=1-T_{h 0}=T_{b} / G
\end{aligned}
$$

where,

$$
G=H_{h r} \gamma / H_{c}=W_{g} c_{g} \Theta_{h} / W_{b} c_{a} \Theta_{c}
$$

$G$ defined by Eq. (12) is called the thermal capacity flow ratio referenced to the blast volume. And in Eqs. (10) and (11), the bar put over $u$ or $T$ indicates the time average value during the corresponding period.

Thermal efficiency of each period is found to be equal each to each as indicated by Eq. (13), when $G=\bar{u}$ or the thermal capacity flow ratio referenced to the air flow rate through the checkerwork, $G^{*}$ defined by Eq. (14), is equal to unity.

$$
\begin{aligned}
\eta & =\eta_{c}=\eta_{h}=T_{b} / G \ldots \ldots . . \\
G^{*} & =G / \bar{u}=W_{g} c_{g} \Theta_{h} / W_{a} c_{a} \Theta_{c}
\end{aligned}
$$

If $G=\bar{u}$, then $W_{g} c_{g} \Theta_{h}$ is equal to $W_{a} c_{a} \Theta_{c}$, and the operation for the case of $G=\bar{u}$ is called as a balanced cycle operation.

\section{Dimensionless Factors of Hot-blast Stoves}

Since the mathematical model given in $I I$. 1 . contains six dimensionless factors, i.e., $T_{b}, H_{c}, H_{h}, A_{c}, A_{h}$ and $\gamma$, which are independent each other, the operating conditions of hot-blast stoves are characterized by the use of these six factors. In terms of these six factors, some other factors such as $G=H_{h} \gamma / H_{c}, h_{h}^{*} / h_{c}^{*}=$ $G \Lambda_{h} / \gamma \Lambda_{c}$ and $\Pi_{c}=H_{c} \Lambda_{c}$ are defined.

The factor $A_{c}$ indicates the effect of heating surface area $A$, and $H_{c}$ indicates the effect of checker mass in the case of stove design. $G, T_{b}$ and $H_{c}$ (or $\Pi_{c}$ ) imply the effect of the flow rate of combustion product, the effect of hot gas temperature and the effect of cycle time, respectively in the case determining the operating conditions.

\section{Effects of Dimensionless Factors}

The effects of each dimensionless factor on the longitudinal distribution of brick temperature and on the thermal efficiencies of each period are investigated by the numerical computations. In the numerical computations the difference equations (15), (16a) and (16b) are used instead of the differential equations (1) and (2).

$$
\begin{aligned}
& t_{k+1, j}=\left(T_{k, j}-t_{k, j}\right) A H \Delta \tau+t_{k, j} \ldots \ldots \ldots \ldots \ldots \ldots \ldots \ldots \ldots \\
& T_{k, j+1}=\left(T_{k, j}-t_{k, j}\right) \exp \left(-\Lambda_{c} \Delta \zeta / u_{k}\right)+\left(t_{k, j}-t_{k, j+1}\right) \\
& \quad \times\left\{1-\exp \left(-\Lambda_{c} \Delta \zeta / u_{k}\right)\right\} u_{k} / \Lambda_{c} \Delta \zeta+t_{k, j+1} \quad(\mathrm{CP}) \ldots(1 \\
& T_{k, j-1}=\left(T_{k, j}-t_{k, j}\right) \exp \left(-\Lambda_{h} \Delta \zeta\right)+\left(t_{k, j}-t_{k, j-1}\right) \\
& \quad \times\left\{1-\exp \left(-\Lambda_{h} \Delta \zeta\right)\right\} / \Lambda_{h} \Delta \zeta+t_{k, j-1} \quad(\mathrm{HP}) \ldots \ldots \ldots
\end{aligned}
$$


where, the suffixes $(k, j)$ indicate the grid of $(\zeta=j \downarrow \zeta$, $\tau=k \Delta \tau$ ). Equations (16a) and (16b) have been derived by the integration of Eq. (2) with respect to $\zeta$ on the basis of the assumption that the longitudinal distribution of brick temperature can be approximated by a straight line within each interval of the adjacent grids.

The time interval and the position interval have been set as $\Delta \tau=\Delta \zeta=1 / 100$ in the following examples, and it has been found that almost the same results are
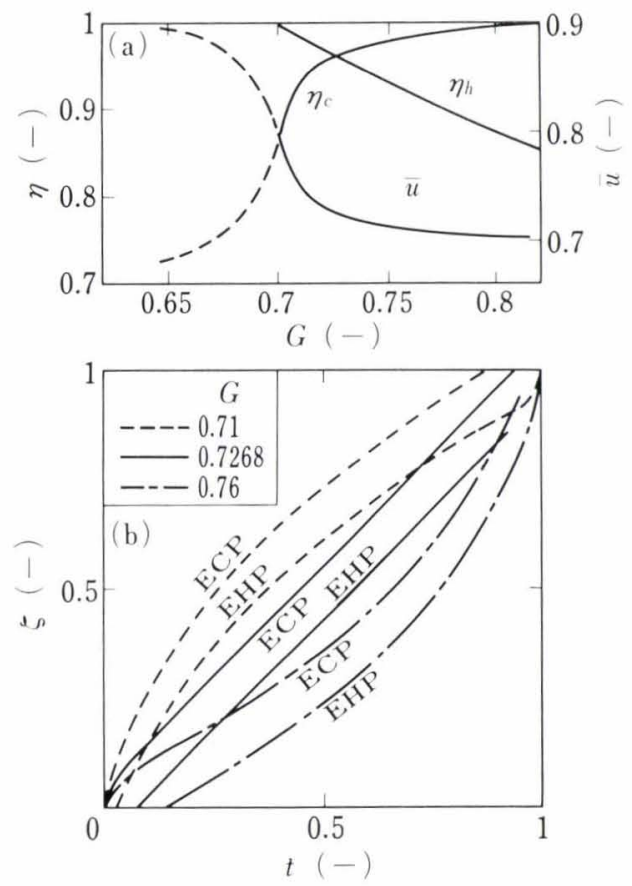

ECP: End of cooling period

EHP: End of heating period

Fig. 2. (a) Effects of $G$ on the thermal efficiency $\left(r_{1}\right)$ and the time-average flow rate of air $(\bar{u})$

(b) Effects of $G$ on the longitudinal distributions of brick temperature
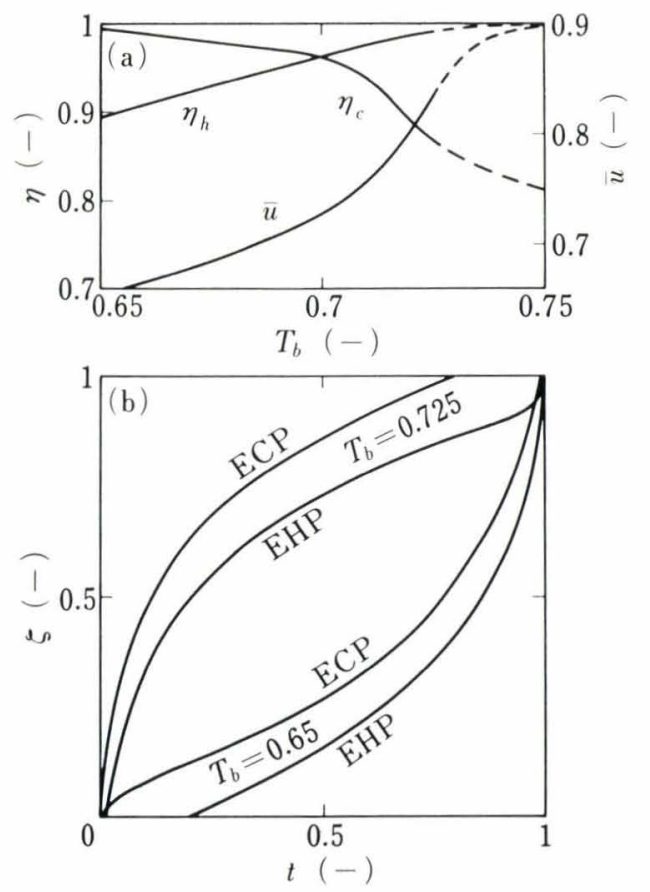

Fig. 3. Effects of nondimensional blast temperature, $T_{b}$
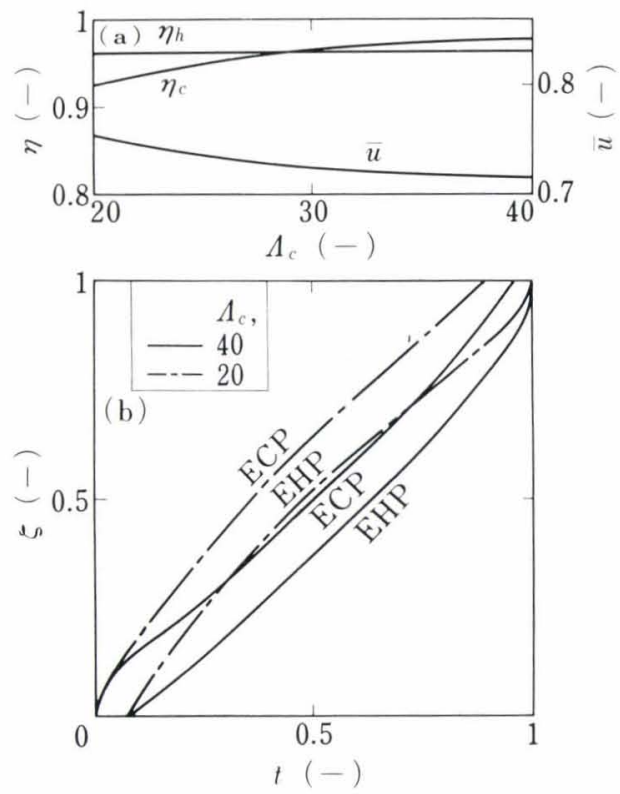

Fig. 4. Effects of modified Stanton number, $A_{c}$
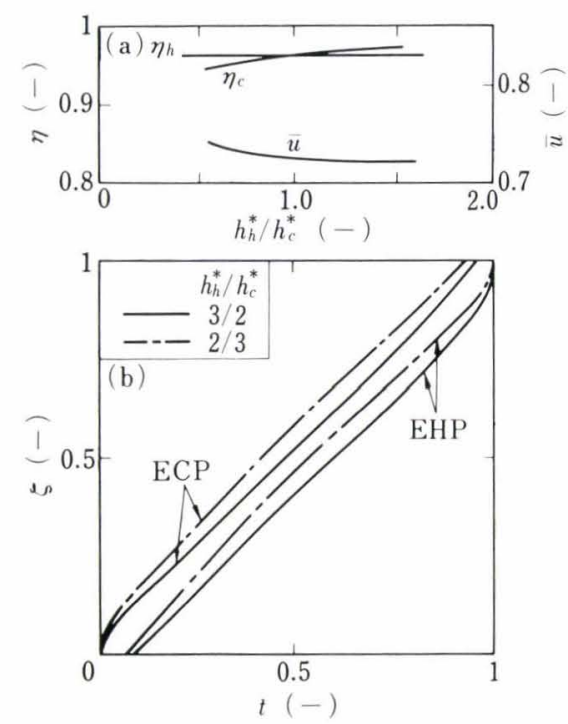

Fig. 5. Effects of the ratio of heat transfer coefficients in both periods, $h_{h}^{*} / h_{e}^{*}$
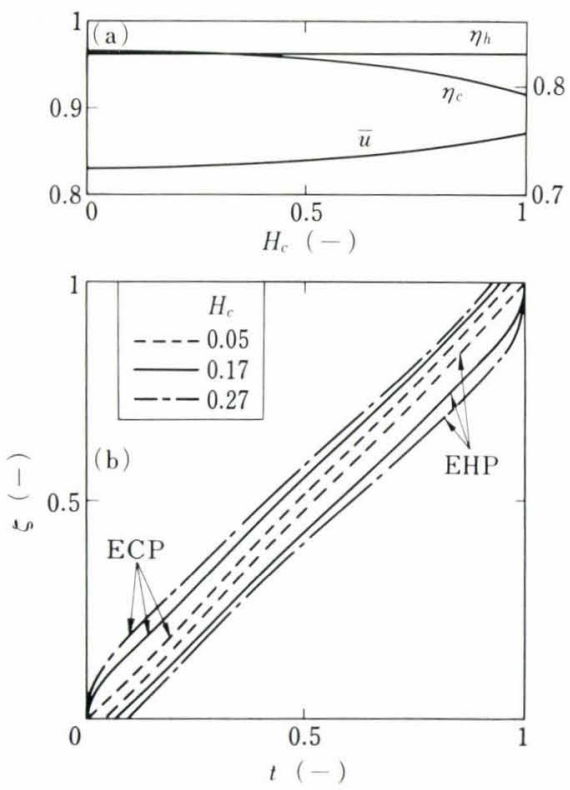

Fig. 6. Effects of $H_{c}$ 
obtained even if $\Delta \tau=\Delta \zeta=1 / 200$.

Examples are shown in Figs. 2 to 6 , whereat each dimensionless factor is perturbed around the values of $T_{b}=0.7, A_{c}=30, h_{h}^{*} / h_{c}^{*}=1.0, H_{c}=0.1699$ and $G=$ 0.7268 . These data have been determined by referring to the practical operating conditions previously reported, ${ }^{11,17)}$ in order to give a numerical example of balanced cycle operations.

Longitudinal distribution of brick temperature is affected by $G^{*}(=G / \bar{u})$ in the similar manner as in the case of a recuperator. That is, when $G^{*}=1$ (i.e., $G=\bar{u}$ ) the curve of temperature $v s$. $\zeta$ becomes linear almost everywhere in the checkerwork. It becomes convex when $G^{*}>1$ (i.e., $G>\bar{u}$ ) and concave when $G^{*}<1$ (i.e., $G<\bar{u}$ ).

Now the flow rate of air through the stove, $\bar{u}$, is regulated by an automatic controller according to the rule given by Eq. (9a), but in Eq. (9a) the value of $T_{c 1}(\tau)$ is unknown. Hence, $G^{*}$ cannot be estimated directly from the given values of operating variables.

Therefore, it may be guessed that among the several effects of the dimensionless factors mentioned above on the stove performance, the effect on $\bar{u}$ is essential.

When $G$ changes around the value corresponding to a balanced cycle, the large decrease in the thermal efficiency of one period may be brought, while a small improvements in the efficiency of another period may be expected, and such behavior is illustrated in Fig. 4(a). From this behavior the balanced cycle operations are found to be near optimal, and, as Green ${ }^{14)}$ has already pointed out, the design calculation may be well done by the use of the solution for only balanced cycle operations.

\section{Approximate Analysis}

\section{Fundamental Oscillations in Balanced Cycle}

Eliminating $T$ from Eqs. (1) and (2), a second order partial differential equation (17) can be obtained.

$$
\frac{\partial^{2} t}{\partial \tau \partial \zeta}+\frac{A}{v}\left(v H_{\partial \zeta}^{\partial t}+\begin{array}{c}
\partial t \\
\partial \tau
\end{array}\right)=0
$$

Among the solutions which satisfy Eq. (17), there exists a solution of a simple form as follows:

$$
t=\left\{\begin{array}{l}
a^{*}\left(\zeta-H_{c} \int_{0}^{\tau} u d \tau\right)+b_{c}^{*} \\
a^{*}\left(\zeta+H_{h} \tau^{\prime}\right)+b_{h}^{*}
\end{array}\right.
$$

where $a^{*}, b_{c}^{*}$ and $b_{h}^{*}$ are arbitrary constants.

This solution may be applicable to the region where the longitudinal distribution of brick temperature becomes linear.

The saw-toothlike oscillations given by Eqs. (18a) and (18b) have been called as the fundamental oscillations of brick temperature. Then the total variation in the brick temperature during a cycle, i.e., the amplitude of the fundamental oscillation, can be expressed as follows:

$$
\Delta t=a^{*} H_{c} \bar{u}=a^{*} H_{h} \gamma
$$

To determine the values of the unknown parameters involved in Eqs. (18a) and (18b), it is usually needed to take into account the behavior of gas and brick temperatures in the vicinity of each end of the checkerwork. Though, in general, the analysis for the end regions becomes more tedious than that of the middle region, the whole problem can be solved quite easily by the aid of the following approximate methods.

\section{Determination of $\Delta t$}

The amount of heats stored and regenerated during a cycle can be determined from a dimensionless equation (20).

$$
Q=\int_{0}^{1}\{t(0, \zeta)-t(1, \zeta)\} d \zeta
$$

From Eq. (20), it may be easily found that $Q$ is equal to the area enclosed by the curves of OABCO in Fig. 7. Then the effective height of the checkerwork, $\Delta \zeta_{e}$, is defined by Eq. (21).

$$
\omega_{e}=Q / Q_{\text {ideal }}
$$

$Q_{\text {ideal }}$ in Eq. (21) means the amount of heat exchanged in the ideal operation where it is assumed that the fundamental oscillation mentioned above is taking place all over the height of the checkerwork, in other words the nonlinear distribution of temperature does not appear even in the entrance regions. Hence,

$$
Q_{\text {ideal }}=\Delta t
$$

Integrating Eqs. (1) and (2) over the interval of $\zeta=0$ to 1 and then using Eqs. (4), (5), (10) and (11), $Q$ can be written as

$$
Q=H_{c} T_{b}
$$

Then, from Eqs. (21) to (23), we have
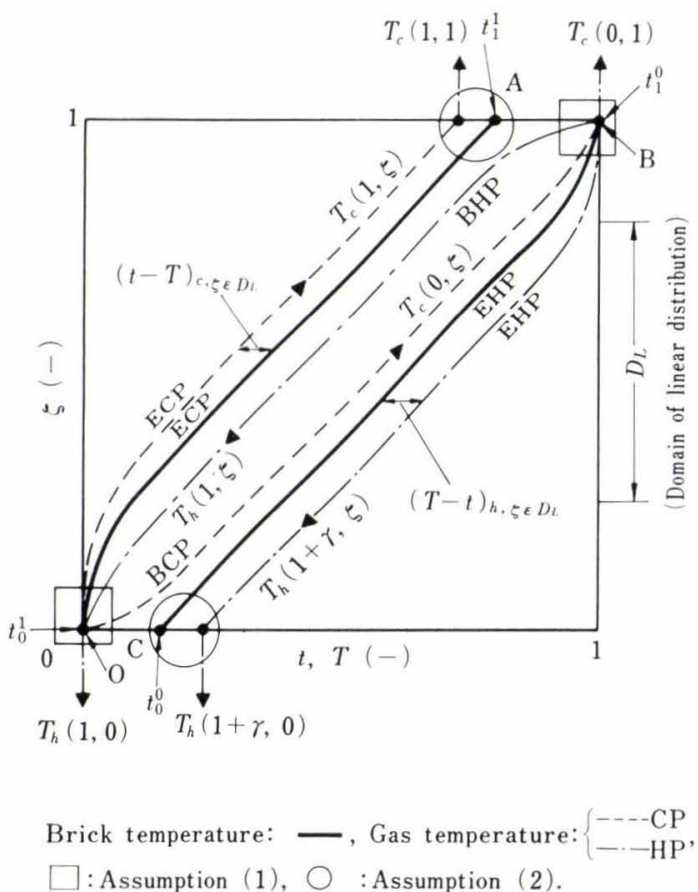

Fig. 7. Assumed pattern of the temperature distributions for the derivation of approximate solutions 


$$
\Delta t=H_{c} T_{b} / \Delta \zeta_{c}
$$

From Eq. (21), it is obvious that $J_{e}$ is equivalent to $K / K_{0}$ introduced by Hausen ${ }^{15)}$ as a correction factor for the overall heat transfer coefficient.

In general, $\Delta \zeta_{e}$ is expressed by Eq. (25).

$$
\zeta_{\zeta}=\frac{\left(T_{b} / \Lambda_{c}\right)\left(\alpha+\bar{u}^{2} / G^{2}-1\right)}{1-\eta+\left(\overline{u t_{c}} / G-\bar{t}_{h}\right)_{\zeta \in D_{L}}}
$$

where, $\alpha$ is defined as follows:

$$
\alpha=1+1 /\left(\gamma h_{h}^{*} / h_{c}^{*}\right)
$$

In the case of a balanced cycle of single-blowing operation where $u \fallingdotseq \bar{u}=G$ and $\left(\bar{t}_{c}-\bar{t}_{h}\right)=0$, Eq. (25) reduces to Eq. (27) which was derived by Hausen. ${ }^{15)}$

$$
\Delta \zeta_{e}=\alpha T_{b} /(1-\eta) \Lambda_{c}
$$

By the use of the new variables $\Phi$ and $\Psi$ defined by Eqs. (28) and (29), respectively, Eq. (27) is transformed into Eq. (27)'.

$$
\begin{array}{r}
\Delta \zeta_{e}=(\Phi-1) / \Psi \ldots \ldots \ldots \ldots \\
\Phi \equiv 1 /(1-\eta)=G /\left(G-T_{b}\right) \ldots \ldots \ldots \\
\Psi \equiv A_{c} / \alpha G=\left(A U / c_{g} W_{q}\right)(1+1 / \gamma)
\end{array}
$$

where, $U$ designates the overall heat transfer coefficient between combustion product and air and is defined as follows:

$$
1 / U=\left(1 / h_{c}^{*} \Theta_{c}+1 / h_{h}^{*} \Theta_{h}\right)\left(\Theta_{c}+\Theta_{h}\right)
$$

It may be seen from Eq. (28) that there is one to one correspondence between $\Phi$ and $\eta$. The closer the thermal efficiency, $\eta$, approaches to unity, the smaller the denominator of Eq. (28) becomes, so that we can use $\Phi$ as the index which magnifies the variations in $\eta$ in its heigher range. On the other hand, the physical meaning of $\Psi$ is analogous to that of $A$.

Substituting Eq. (27) into Eq. (24), Eq. (31) can be obtained.

$$
\Delta t=H_{c} A_{c}(1-\eta) / \alpha
$$

or by using $\Phi$ and $\Psi$,

$$
\Delta t / G H_{c}=\Psi / \Phi
$$

From Eqs. (1), (2), (18), (19) and (31), ( $\partial t / \partial \zeta)$ and $(\mathcal{T}-t)$ relevant to the fundamental oscillation are also obtained as follows:

$$
\begin{aligned}
& (\partial t / \partial \zeta)_{\zeta \in D_{L}}=(1-\eta) \Lambda_{c} / \alpha G=\Psi / \Phi \ldots \ldots \ldots \\
& (T-t)_{\zeta \in D_{L}}= \begin{cases}-(1-\eta) / \alpha & (\mathrm{CP}) \ldots \ldots \\
(1-\eta)(\alpha-1) / \alpha & (\mathrm{HP}) \ldots \ldots\end{cases}
\end{aligned}
$$

Therefore, if the heat transfer coefficient, $h^{*}$, can be estimated, it becomes possible to calculate $\Delta t,(\partial t / \partial \zeta)$ and $(T-t)$ from the design and operating conditions.

\section{Determination of Longitudinal Distribution of Brick Temperature}

In order to determine the longitudinal distribution of brick temperature under the given operating conditions, it is necessary to consider the behavior of brick temperature in each entrance region.

Hausen ${ }^{10)}$ treated approximately the end region of a regenerator and obtained a set of solution equations as shown later. Hausen's solution is not so simple that when the average flow rate of air in the cooling period is not given as usual, it is difficult to solve the whole problem of hot-blast stoves by the use of his solution.

The authors' method shown below is based on the following assumptions:

(1) At the beginning of each period, the exit gas temperature is nearly equal to the inlet temperature of the gas flowed during the preceding period, since the temperature of brick in the region near the gas outlet has become equal to the inlet gas temperature of the preceding period.

(2) At the end of each period, the longitudinal distributions of gas and brick temperatures in the region near the gas exit become almost as linear as the distributions in the middle region of the checkerwork.

The assumptions are also illustrated in Fig. 7.

The first assumption is expressed by Eqs. (34a) and (34b).

$$
\begin{aligned}
& T_{c 1}(\tau=0) \fallingdotseq 1 \\
& T_{h 0}(\tau=1) \fallingdotseq 0 .
\end{aligned}
$$

Considering the second assumption, Eqs. (35a) and (35b) can be obtained from Eqs. (2), (33a), and (33b).

$$
\begin{aligned}
T_{c 1}(\tau=1) & \fallingdotseq t_{1}^{1}-(1-\eta) / \alpha \ldots \ldots \\
T_{h 0}(\tau=1+\gamma) & \fallingdotseq t_{0}^{0}+(1-\eta)(\alpha-1) / \alpha
\end{aligned}
$$

Furthermore from the second assumption, a simple geometrical relation as expressed by Eq. (36) can be found between $t_{0}^{0}$ and $t_{1}^{1}$.

$$
t_{0}^{0}=t_{1}^{1}+\Delta t-(\partial t / \partial)_{\zeta \epsilon D_{L}}
$$

where, the definitions of $T_{c 1}(\tau=0), T_{c 1}(\tau=1), T_{h 0}(\tau$ $=1), T_{h 0}(\tau=1+\gamma), t_{1}^{1}$ and $t_{0}^{0}$ are illustrated in Fig. 7 .

If the above assumptions are reasonable, the longitudinal distribution of temperature at the end of each period may be obtained as a straight line drawn through the point $\left(1, t_{1}^{1}\right)$ or the point $\left(0, t_{0}^{0}\right)$ with the slope of $(\partial t / \partial \zeta)=(1-\eta) \Lambda_{c} / \alpha G$. Therefore it becomes the aim of the approximate analysis to express $t_{1}^{1}$ and $t_{0}^{0}$ by the simple functions of operating conditions.

\section{Approximate Solution A}

When the average temperature of exit gas, $T_{c 1}$ and $T_{h 0}$, can be written by the arithmetic mean of the initial and final temperatures of each period, Eqs. (37) and (38) may be obtained from Eqs. (34) and (35).

$$
\begin{aligned}
& \overline{\mathcal{T}}_{c 1}=\left\{1+t_{1}^{1}-(1-\eta) / \alpha\right\} / 2 \\
& \overline{\mathcal{T}}_{h 0}=\left\{t_{0}^{0}+(1-\eta)(\alpha-1) / \alpha\right\} / 2
\end{aligned}
$$

Since the left hand sides of Eqs. (37) and (38) have been known from the overall heat balance Eqs. (10) and (11), $t_{1}^{1}$ and $t_{0}^{0}$ can be given by Eqs. (39) and (40), respectively. 


$$
\begin{aligned}
& t_{1}^{1}=1-(2-1 / \alpha)(1-\eta) \\
& t_{0}^{0}=(1+1 / \alpha)(1-\eta)
\end{aligned}
$$

\section{Approximate Solution B}

In order to get more accurate expressions for $t_{1}^{1}$ and $t_{0}^{0}$, the relations between $t_{1}^{1}$ and $T_{c 1}$ or $t_{0}^{0}$ and $T_{h 0}$ should be determined on the basis of Eq. (1). For the facility of the analysis the periodic variations in $t_{1}$ and in $t_{0}$ are approximately expressed by the following quadratic equations and in these equations four unknown parameters are involved.

$$
\begin{aligned}
& t_{1}=1-b \tau-a \tau^{2} \\
& t_{0}=b^{\prime} \tau^{\prime}+a^{\prime} \tau^{\prime 2}
\end{aligned}
$$

where, $\tau^{\prime}$ is the dimensionless elapsed time from the beginning of heating period.

Following two equations at $\zeta=1$ and $\zeta=0$ respectively are obtained from Eq. (1).

$$
\begin{aligned}
& T_{c 1}=t_{1}+\left(1 / \Pi_{c}\right)\left(d t_{1} / d \tau\right) . \\
& T_{h 0}=t_{0}+\left(1 / \Pi_{h}\right)\left(d t_{0} / d \tau^{\prime}\right)
\end{aligned}
$$

Substituting Eq. (41) into Eq. (43) and also substituting Eq. (42) into Eq. (44), and then integrating Eq. (43) over the interval from $\tau=0$ to 1 and Eq. (44) over $\tau^{\prime}=0$ to $\gamma$, Eqs. (45) and (46) can be obtained, respectively.

$$
\begin{aligned}
\bar{T}_{c 1}=\eta=1- & \left(1 / \Pi_{c}+2 / 3\right)(a+b)+(2 a+b) / 6 . \\
\bar{T}_{h 0}=1-\eta= & \left(1 / \gamma \Pi_{h}+2 / 3\right)\left(a^{\prime} \gamma^{2}+b^{\prime} \gamma\right) \\
& +\left(2 a^{\prime} \gamma^{2}+b^{\prime} \gamma\right) / 6 \ldots \ldots \ldots \ldots \ldots \ldots . .
\end{aligned}
$$

On the other hand, Eqs. (47) and (48) can be written from the second assumption mentioned above, since the time derivative of brick temperature in the middle region of the checkerwork can be written from Eqs. (1), (31) and (33) as follows:

$$
\begin{aligned}
(\partial t / \partial \tau)_{\zeta \in D_{L}} & = \begin{cases}-\Delta t & (\mathrm{CP}) \\
\Delta t / \gamma & (\mathrm{HP})\end{cases} \\
\left(d t_{1} / d \tau\right)_{\tau=1} & =-(2 a+b)=-\Delta t . \\
\left(d t_{0} / d \tau^{\prime}\right)_{\tau^{\prime}=\gamma} & =2 a^{\prime} \gamma+b^{\prime}=\Delta t / \gamma
\end{aligned}
$$

The unknown parameters, $a, b, a^{\prime}$ and $b^{\prime}$, can be obtained by solving Eqs. (45) to (48) simultaneously, and substituting them into Eqs. (41) and (42), we have the time variations in $t_{1}$ and $t_{0}$. Hence,

$$
\begin{aligned}
& t_{1}^{1}=1-(1-\eta+\Delta t / 6) /\left(1 / \Pi_{c}+2 / 3\right) \\
& t_{0}^{0}=(1-\eta+\Delta t / 6) /\left(1 / \gamma \Pi_{h}+2 / 3\right)
\end{aligned}
$$

\section{Necessary Conditions for Balanced Cycle Operation}

In the above analysis it has been assumed that the operating variables are set so as to give a balanced cycle operation, but it is not clarified that under what conditions the stove cycle becomes a balanced cycle. So the necessary conditions which should be satisfied by the five nondimensional factors, $\Lambda_{c}, H_{c}, G, T_{b}$ and $\alpha$ (or $A_{c}, \Pi_{c}, G, \eta$ and $\alpha$ ), to realize a balanced cycle can be derived as follows.

When solution A (Eqs. (39) and (40)) and Eq. (31) are substituted into Eq. (36), Eq. (51) or (51)' can be obtained as the necessary condition for a balanced cycle.

$$
\begin{array}{ll}
\Lambda_{c}=G\left[\alpha\{1 /(1-\eta)-3\}+\Pi_{c}\right] & (\text { solution A) ...(51) } \\
\Psi-\Phi=\Pi_{c} / \alpha-3 & (\text { solution A)...(51)' }
\end{array}
$$

In the same manner, Eqs. (52) or (52)' can be obtained from solution B (Eqs. (49) and (50)).

$$
\begin{aligned}
& \Lambda_{c}=G\left[\alpha\{1 /(1-\eta)-3 X\}+\Pi_{c}(1-X / 2)\right] \\
& \quad(\text { solution B). } \\
& \Psi-\Phi=\left(\Pi_{c} / \alpha\right)(1-X / 2)-3 X \quad \text { (solution B).. }
\end{aligned}
$$$$
\text { (solution B) ...(52) }
$$

where, $X$ is defined by Eq. (53) and varies within the range of $0<X<1$.

$$
X=1 /\left(3 / \Pi_{c}+2\right)+1 /\left\{3(\alpha-1) / \Pi_{c}+2\right\}
$$

Equation (51) or (52) is a constraint for the values of five dimensionless variables mentioned above. Therefore, by substituting the data of four variables into these equations the value of remaining one variable can be determined.

Now, comparing Eq. (51)' with Eq. (52)', it may be found that the right hand sides of both equations give considerably different values and that sometimes the values of the right hand side of Eq. (51)' are two times larger than those of Eq. (52)'. However, it can be shown that the difference mentioned above is not so essential in the calculation of $A_{c}, T_{b}$ and $G$. To show this, the right hand side of Eq. (51)' or $(52)^{\prime}$ is replaced with $f$.

$$
\Psi-\Phi=f\left(\Pi_{c}, \alpha\right)
$$

Then, the sensitivity coefficients concerning the change in $f$ are derived as follows:

$$
\begin{aligned}
& \mu_{A} \equiv\left(f / A_{c}\right)\left(\partial \Lambda_{c} / \partial f\right)=f / \Psi \\
& \mu_{T} \equiv\left(f / \mathcal{T}_{b}\right)\left(\partial T_{b} / \partial f\right)=-f \Phi /(\Phi-1)(\Phi-f)^{2} \ldots \\
& \mu_{G} \equiv(f / G)(\partial G / \partial f)=f /\left(\Phi^{2}-\Phi-\Psi\right) \ldots \ldots \ldots \ldots \ldots \ldots \ldots \ldots \ldots \ldots \ldots \ldots \ldots \ldots \ldots
\end{aligned}
$$

Since $f$ is much smaller than $\Phi$ and $\Psi$ in the practical cases, these sensitivity coefficients become very small and so the variations in $f$ cause the little changes in $A_{c}, T_{b}$ and $G$.

When the value of $\Pi_{c}$ (or $H_{c}$ ) is determined from Eq. (51) or (52), the results obtained from these equations differ considerably each other. However, the effect of $\Pi_{c}$ (or $H_{c}$ ) on $\bar{u}$ is not so large as already shown in Fig. 6 (a) and $\Pi_{c}$ (or $H_{c}$ ) can be varied under the condition of $G \fallingdotseq \bar{u}$ rather widely around the value which gives the exact balanced cycle.

Hence, both criterion equations (Eqs. (51) and (52)) may be used for the practical calculations but it is required to take account of the stove characteristics mentioned above and the accuracy of each equaiion as shown later.

In addition to Eq. (51) or (52), the other necessary conditions expressed in the form of inequality must be considered. The conditions are

$$
\begin{aligned}
J_{\zeta} & =\alpha T_{b} /(1-\eta) \Lambda_{c} \leq 1 \\
\Delta t & =H_{c} \Lambda_{c}(1-\eta) / \alpha<1
\end{aligned}
$$




$$
u(\tau=1)=T_{b} /\left[t_{1}^{1}-(1-\eta) / \alpha\right] \leq 1
$$

5. Comparison between Approximate Solutions and $\mathrm{Nu}$ merical Solution

In order to examine the validity of the approximate solutions obtained above, the results calculated by using the approximate computations (A and $\mathrm{B}$ ) and those determined by numerical computations are compared in Table 1. The values of $G$ given in Table 1 have been determined by substituting the other parameters into Eq. (51) or (52) which is the necessary condition for the balanced cycle operation. The data obtained in this way have been examined by using the inequality conditions (58) to (60) and supplied into the computer to perform the numerical computations. Furthermore, the longitudinal distribution of brick temperature and the values of $\bar{u}$ have been determined, and these results have been compared with those obtained from the approximate solutions.

If the approximate solution is valid, the values of $\bar{u}$ obtained by numerical computations $\left(\bar{u}_{\text {num }}\right)$ should be equal to the values of $G\left(=\bar{u}_{\text {appr }}\right)$ obtained from the necessary condition. In addition, the values of $t_{1}^{1}, t_{0}^{0}$ and $\Delta t$ calculated by both approximate and numerical solutions should be equal each to each. Table 1 shows that these values are in fairly good agreements. In spite of its simplicity, the approximate solution A gives good results in most cases. However, in the cases of runs 7 to 9 , the differences between the estimated $\bar{u}_{\text {appr }}$ and the computed $\bar{u}_{\text {num }}$ become larger. But, in these cases the approximate solution B gives still accurate results. In regard to $t_{1}^{1}$ and $t_{0}^{0}$ the approximate solution $\mathrm{B}$ is more close to the numerical solutions.

Figure 8 shows the comparisons about the longitudinal distributions of brick temperature in the case of runs 1 and 4 . In the other cases except runs 7 and 9 similar agreements have been obtained. However, in the case of run 7 or 9 whereat the amplitude, $\Delta t$, is much larger than those of the other cases, the brick temperature distributions obtained from the approximate solution $\mathrm{A}$ deviate from the numerical results.

Now, it is interesting to compare the results of the present solutions $\mathrm{A}$ and $\mathrm{B}$ with the approximate solution previously reported by Hausen. ${ }^{10) *}$ Table 2 shows the results of $t_{1}^{1}$ calculated from three kinds of approximate solutions. It is found that though Hausen's solution gives a good results, the approximate solution $\mathrm{B}$ which has been derived without such complicated analysis as Hausen's method gives more accurate results. Although the errors accompanied with the results of approximate solution $\mathrm{A}$ are at most $1.5 \%$ in regard to $t_{1}^{1}$, the magnitude of these errors is not so large.

In the practical problems it is important to estimate $\bar{u}$ or the thermal efficiency $\eta=\mathcal{T}_{b} / \bar{u}=T_{b} / G$. From this point of view, both solutions $\mathrm{A}$ and $\mathrm{B}$ are considered to be successful in most practical cases.

Furthermore, though in the cases of runs 1 to 6 given in Table 1 the value of $u$ changes up to $31 \%$ around its average $\bar{u}$, the approximate solutions which

Table 1. Comparison of approximate solutions with numerical solution (A: Approximate solution A, B: Approximate solution B)

\begin{tabular}{|c|c|c|c|c|c|c|c|c|c|c|c|c|c|}
\hline Run & $T_{b}$ & $A_{c}$ & $H_{c}$ & $G\left(=\bar{u}_{\mathrm{appr}}\right) *$ & & $\tilde{u}_{\text {num }}$ & $\varepsilon^{* *}$ & $t_{1}^{1}$, appr & $t_{1}^{1}$, num & $t_{0}^{0}$, appr & $t_{0}^{0}$, пит & $\Delta t_{\mathrm{appr}}$ & $\Delta t_{\mathrm{num}}$ \\
\hline \multirow{2}{*}{1} & \multirow{2}{*}{0.7} & \multirow{2}{*}{9} & \multirow{2}{*}{0.3960} & A 0.7980 & A & 0.8050 & 0.88 & 0.836 & 0.814 & 0.205 & 0.209 & 0.292 & 0.305 \\
\hline & & & & В $\quad 0.7949$ & B & 0.8084 & 1.70 & 0.822 & 0.809 & 0.209 & 0.204 & 0.284 & 0.305 \\
\hline \multirow{2}{*}{2} & \multirow{2}{*}{0.7} & \multirow{2}{*}{12} & \multirow{2}{*}{0.3146} & A 0.7710 & A & 0.7721 & 0.14 & 0.877 & 0.862 & 0.154 & 0.161 & 0.232 & 0.238 \\
\hline & & & & B 0.7687 & B & 0.7742 & 0.72 & 0.862 & 0.859 & 0.161 & 0.156 & 0.225 & 0.238 \\
\hline \multirow{2}{*}{3} & \multirow{2}{*}{0.7} & \multirow{2}{*}{15} & \multirow{2}{*}{0.2665} & A 0.7557 & A & 0.7552 & -0.07 & 0.902 & 0.889 & 0.123 & 0.131 & 0.197 & 0.200 \\
\hline & & & & В 0.7539 & B & 0.7565 & 0.34 & 0.886 & 0.886 & 0.132 & 0.129 & 0.191 & 0.200 \\
\hline \multirow{2}{*}{4} & \multirow{2}{*}{0.7} & \multirow{2}{*}{30} & \multirow{2}{*}{0.1699} & A 0.7268 & A & 0.7258 & -0.14 & 0.951 & 0.938 & 0.062 & 0.073 & 0.125 & 0.125 \\
\hline & & & & B 0.7260 & B & 0.7260 & 0.00 & 0.934 & 0.938 & 0.074 & 0.072 & 0.122 & 0.125 \\
\hline \multirow{2}{*}{5} & \multirow{2}{*}{0.8} & \multirow{2}{*}{30} & \multirow{2}{*}{0.1610} & A 0.8352 & A & 0.8339 & -0.16 & 0.944 & 0.931 & 0.070 & 0.081 & 0.136 & 0.136 \\
\hline & & & & B 0.8340 & B & 0.8343 & 0.04 & 0.927 & 0.931 & 0.082 & 0.080 & 0.131 & 0.136 \\
\hline \multirow{2}{*}{6} & \multirow{2}{*}{0.7} & \multirow{2}{*}{60} & \multirow{2}{*}{0.1164} & A 0.7131 & A & 0.7130 & -0.01 & 0.976 & 0.963 & 0.031 & 0.042 & 0.086 & 0.085 \\
\hline & & & & В 0.7127 & B & 0.7132 & 0.26 & 0.960 & 0.963 & 0.044 & 0.042 & 0.083 & 0.085 \\
\hline \multirow{2}{*}{7} & \multirow{2}{*}{0.8} & \multirow{2}{*}{26.3} & \multirow{2}{*}{0.6562} & A 0.8762 & A & 0.8368 & -4.50 & 0.884 & 0.841 & 0.145 & 0.308 & 1.000 & 0.751 \\
\hline & & & & В 0.8489 & B & 0.8524 & 0.42 & 0.670 & 0.780 & 0.343 & 0.225 & 0.663 & 0.751 \\
\hline \multirow{2}{*}{8} & \multirow{2}{*}{0.8} & \multirow{2}{*}{25.1} & \multirow{2}{*}{0.0010} & A 0.8364 & A & 0.8532 & 2.01 & 0.945 & 0.978 & 0.071 & 0.040 & 0.0008 & 0.0006 \\
\hline & & & & B 0.8423 & B & 0.8543 & 1.43 & 0.999 & 0.978 & 0.0 & 0.042 & 0.0008 & 0.0007 \\
\hline \multirow{2}{*}{9} & 0.8 & 2.57 & A 0.4661 & & A & 0.8385 & -2.17 & 0.869 & 0.911 & 0.200 & 0.111 & 0.532 & 0.424 \\
\hline & 0.8 & 2.51 & В 0.8754 & $0.85 / 1$ & B & 0.8558 & -0.15 & 0.672 & 0.685 & 0.339 & 0.342 & 0.9997 & 0.937 \\
\hline
\end{tabular}

* Data have been determined by using Eq. (51) or (52). ** $\varepsilon \equiv 100 \times\left(\bar{u}_{\mathrm{num}}-\bar{u}_{\mathrm{appr}}\right) / \bar{u}_{\mathrm{appr}}(\%)$.

* Hausen's approximate solution regarding $t_{1}^{1}$ consists of the following set of equations.

$$
\begin{array}{ll}
t_{1}^{1}=1-\left(1-T_{c 1}^{0}\right)\left[1+\beta\left\{\left(1-2 n / \Pi_{c}\right)\left(1-1 / \Pi_{c}\right)+n\right\}\right] & \beta \equiv(\alpha-1) /\left[\left(1-2 n / \Pi_{c}\right)\left\{1+(\sigma-1) / \Pi_{c}\right\}+n\right] \\
T_{c 1}=1-\left(1-T_{b} / \bar{u}\right) /\{1+\beta(0.5+n / 3)\} & \sigma \equiv \exp \left(\Pi_{h} \gamma\right), n \equiv 0.0065 \Pi_{c}^{2.5} \quad(\text { for the case of } G=\bar{u})
\end{array}
$$


have been derived on the basis of the assumption $u(\tau) \fallingdotseq \bar{u}$ show good agreements with the numerical

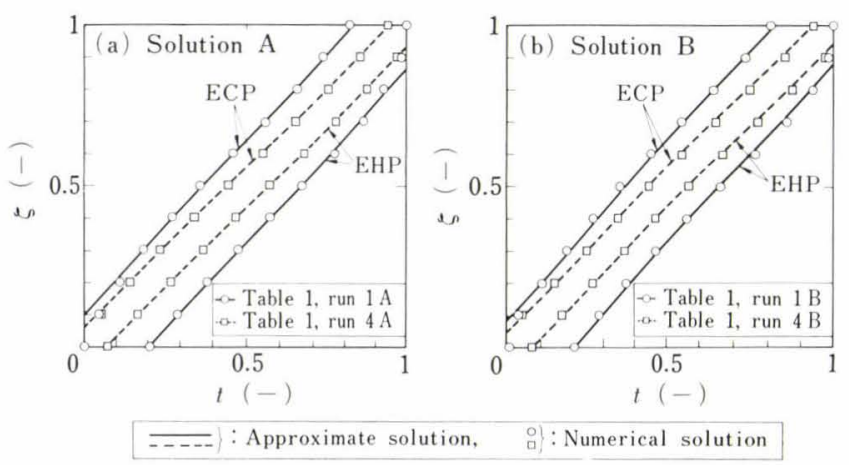

Fig. 8. Comparison between brick temperatures obtained by approximate analyses and those by numerical computations

Table 2. Comparison of three approximate solutions for $t_{1}^{1}$

\begin{tabular}{|c|c|c|c|c|c|c|c|c|}
\hline \multirow{2}{*}{ Run } & \multicolumn{4}{|c|}{ Operating conditions } & \multirow{2}{*}{$\begin{array}{l}\text { Numeri- } \\
\text { cal } \\
\text { solution }\end{array}$} & \multicolumn{3}{|c|}{ Approximate solutions } \\
\hline & $T_{b}$ & $\Lambda_{c}$ & $H_{c}$ & $G$ & & A & B & $\begin{array}{c}\text { Hau- } \\
\text { sen's }\end{array}$ \\
\hline 1 & 0.7 & 9 & 0.3960 & 0.7980 & 0.814 & $\begin{array}{l}0.836 \\
(2.7)^{*}\end{array}$ & $\begin{array}{l}0.819 \\
(0.6)\end{array}$ & $\begin{array}{c}0.838 \\
(2.9)\end{array}$ \\
\hline 2 & 0.7 & 12 & 0.3146 & 0.7710 & 0.862 & $\begin{array}{l}0.877 \\
(1.7)\end{array}$ & $\begin{array}{l}0.860 \\
(0.3)\end{array}$ & $\begin{array}{l}0.875 \\
(1.5)\end{array}$ \\
\hline 3 & 0.7 & 15 & 0.2665 & 0.7557 & 0.889 & $\begin{array}{l}0.902 \\
(1.5)\end{array}$ & $\begin{array}{l}0.883 \\
(0.6)\end{array}$ & $\begin{array}{l}0.897 \\
(0.9)\end{array}$ \\
\hline 4 & 0.7 & 30 & 0.1699 & 0.7268 & 0.938 & $\begin{array}{l}0.951 \\
(1.4)\end{array}$ & $\begin{array}{l}0.933 \\
(0.6)\end{array}$ & $\begin{array}{c}0.942 \\
(0.5)\end{array}$ \\
\hline 5 & 0.8 & 30 & 0.1610 & 0.8352 & 0.931 & $\begin{array}{l}0.944 \\
(1.4)\end{array}$ & $\begin{array}{l}0.926 \\
(0.5)\end{array}$ & $\begin{array}{l}0.937 \\
(0.6)\end{array}$ \\
\hline 6 & 0.7 & 60 & 0.1164 & 0.7131 & 0.963 & $\begin{array}{c}0.976 \\
(1.4)\end{array}$ & $\begin{array}{c}0.960 \\
(0.3)\end{array}$ & $\begin{array}{r}0.967 \\
(0.5)\end{array}$ \\
\hline
\end{tabular}

* The values in the parentheses mean the accuracy of estimation in $\%$.

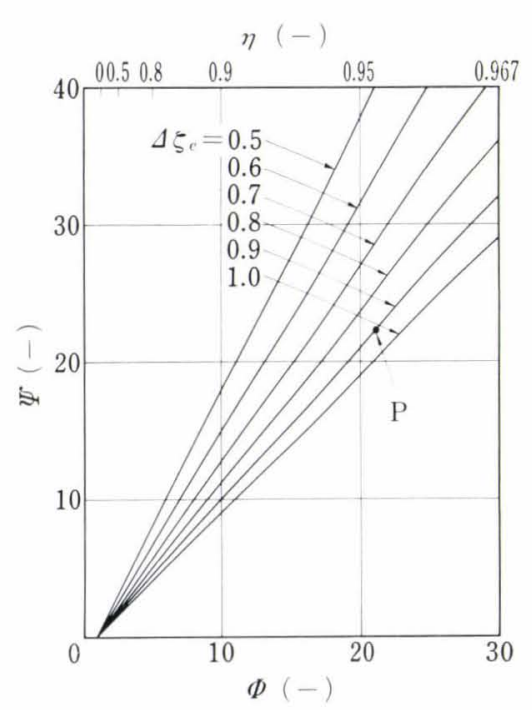

Fig. 9. Diagram for determining the values of effective height $\Delta_{\zeta}$ results. Therefore, it is expected that the approximate solutions can be applied to the problems where the flow rate of hot gas varies with time. For such cases, the average value of the flow rate of hot gas should be used.

\section{Method of Stove Calculation}

\section{1. $\Phi \_\Psi$ Diagrams}

On the basis of the approximate analysis mentioned above, several nomograms (Figs. 9 to 12) for the stove calculation are drawn on the $\Phi-\Psi$ plane. Figure 9 is drawn by the use of Eq. (27)' for the effective height of the checkerwork. For $\Delta t$ and $\left(\partial t / \delta \zeta_{\zeta}\right) \epsilon_{L}$ Fig. 10 is obtained from Eqs. (31)' and (32).

Relation between $\Phi$ and $\Psi$ for a given value of $\Pi_{c} / \alpha$ is determined by the necessary condition for balanced cycle operations. Such relations are plotted in Fig. 11 for the case of approximate solution B (Eq. $\left.(52)^{\prime}\right)$. For the case of approximate solution A, if the values of $\Pi_{c} / \alpha$ in Fig. 11 were reduced to half, almost similar lines could be obtained. When the operating conditions satisfy the necessary condition Eq. (52)' in solution B, Fig. 12 gives the value of $\Delta t$.

Now, as shown in Fig. 12, the existence region of a balanced cycle operation is determined on the basis of the inequalities (58) to (60).

When an operating point $\mathrm{P}(\Phi, \Psi)$ exists in the existence region, it is possible to realize a balanced cycle by regulating the values of operating variables so as to satisfy the necessary condition Eq. (52)' or $(51)^{\prime}$. For example, the operating points corresponding to the operating conditions given in Figs. 2 to 5 and Table 1 are also plotted in Fig. 12.

By using these nomograms, the stove problems can be understood and solved easily. As an example, if $T_{b}=0.8, \eta=0.95(: \Phi=21.25), \quad \zeta_{e}=0.9$ and $\gamma=2$ are specified beforehand and also the values of $h_{c}^{*}$ and

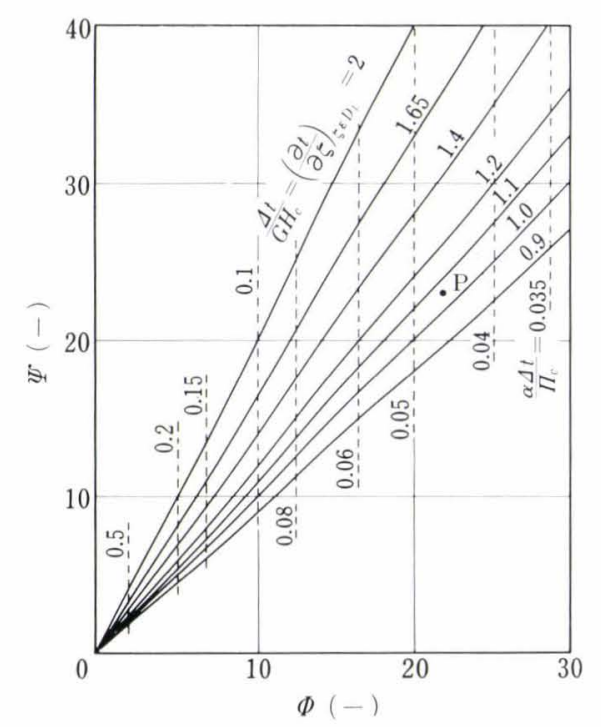

Fig. 10. Diagram for determining the values of brick-temperature amplitude $\Delta t$ and the longitudinal gradient of brick temperature $\partial t / \partial \zeta$

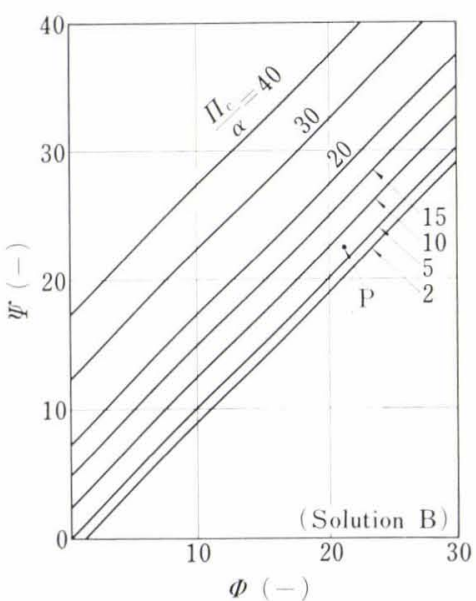

Fig. 11. Necessary value of $\Pi_{c} / \alpha$ to realize a balanced cycle 


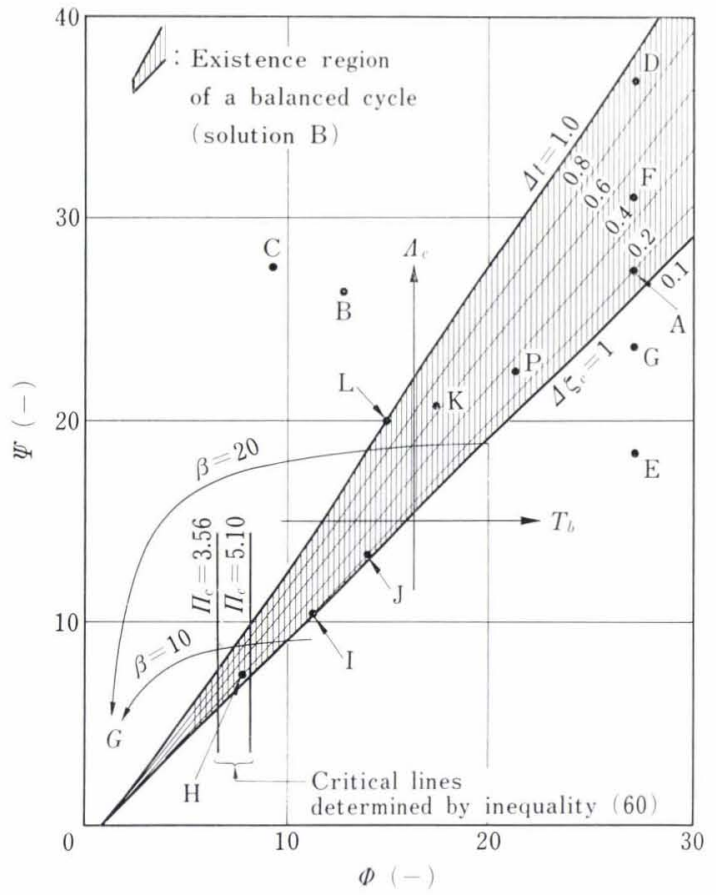
A: Fig. 2, $G=0.7268$
G: Fig. $5, h_{h}^{*} / h_{c}^{*}=2 / 3$
B: Fig. $2, G=0.76$
$\mathrm{H}$ : Table 1 , run $1 \mathrm{~B}$
C: Fig. 3, $T_{b}=0.65$
I: Table 1, run 2 B
D: Fig. $4, \Lambda_{c}=40$
$\mathrm{J}$ : Table 1 , run $3 \mathrm{~B}$
E: Fig. $4, \Lambda_{c}=20$
$\mathrm{K}$ : Table 1 , run $7 \mathrm{~B}$
F: Fig. $5, h_{h}^{*} / h_{c}^{*}=3 / 2$
L: Table 1 , run 9

Fig. 12. Existence region of balanced cycle, values of $\Delta t$ and the loci of operating point when $T_{b}, \Lambda_{c}$ or $G$ is increased

$h_{h}^{*}$ are known $\left(e . g ., h_{h}^{*} / h_{c}^{*}=1\right)$, the position of the operating point is determined from Fig. 9 as shown by the point $\mathrm{P}$, and this operating point is also shown in Figs. 10 to 12 . Then, it is easy to obtain the values $\Pi_{c}=11.25, G=0.84, A_{c}=28.35$ and $\Delta t=$ 0.37 applying these figures with Eqs. (26), (28), and (29).

\section{Near Optimal Solution of Stove Problem}

The effects of the changes in operating conditions can be discussed by the use of $\Phi-\Psi$ diagram. It is found from the definition of $\Phi_{c}$ and $\Psi$ that when $T_{b}$ is varied without changing $\Lambda_{c}$ and $G$, the operating point moves parallel to $\Phi$ axis and that it moves parallel to $\Psi$ axis when $A_{c}$ is varied but the other parameters are kept constant. In the case when $G$ is changed, the locus of an operating point is given by Eq. (61) which is derived by eliminating $G$ from Eqs. (28) and (29).

$$
\Psi=\beta(1-1 / \Phi)
$$

where,

$$
\beta \equiv A_{c} / \alpha T_{b}
$$

Examples of the three cases mentioned above are also shown in Fig. 12. It may be found from Fig. 12 that when the operating point is located on the line of $\Delta \zeta_{e}=1$, a maximum blast temperature is obtained for a given value of the heating surface area, $A$ (or

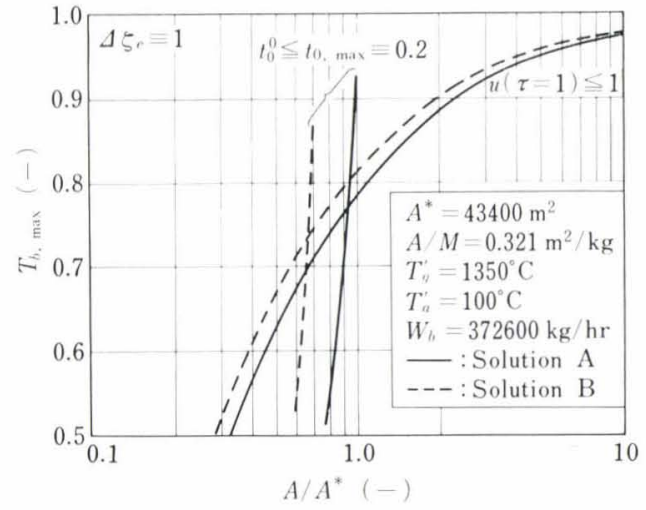

Fig. 13. Available maximum blast temperature corresponding to a given value of $A$, where $h_{c}^{*}=6.0+0.738$ $\times 10^{-4} W_{a}$ and $h_{h}^{*}=8.0+0.765 \times 10^{-4} W_{g}{ }^{16)}$ are applied

of $\Psi$ ), and $A$ has a minimum for a given value of thermal efficiency (or of $\Phi$ ). Furthermore, $G$ or the flow rate of combustion product, $W_{y}$, has also a minimum on the line of $\zeta_{\zeta_{e}}=1$, when $\beta$ is maintained at a constant value. In order to bring the operating point nearer to the line of $\rfloor_{e}=1, \Pi_{c}$ or the cycle time $(1+\gamma) \theta_{c}$ should be reduced as seen from Fig. 11 .

It may be concluded that for the problems determining the design or operating variables the equation expressing the condition of $J_{\zeta_{e}}=1$ should be used as the equation for the near optimal solution. When the cycle time is kept at the minimum value, Eq. (51) or (52) should be applied instead of the condition of \lrcorner$\zeta_{e}=1$.

Modern blast furnace operation needs a heigher blast temperature. For such cases, corresponding to the maximum flow rate of hot gas within the constraints of $G<1, u(\tau=1)<1$ and $t_{0}^{0}<t_{0 \max }$, the minimum value of the necessary area of heating surface may be determined. Where, $t_{0 \max }$ designates the maximum allowable temperature of brick at the lower end of the checkerwork and it may be determined from a stand point of the maintenance of grate.

From the above inequality constraints and Eqs. (50) and (52), the relations between $T_{b \max }$ and $A$ can be obtained. Calculated examples are shown in Fig. 13. If the result is combined with some economical criterions, an optimal solution for the heating surface area may be obtained. Since the solution obtained here is included in the category of balanced cycle, it is not an exact optimum but a near optimal solution.

\section{Conclusion}

The problem of hot-blast stoves is a two point boundary value problem of partial differential equations where the flow rate of air in the cooling period is unknown. In this paper, in order to obtain a simple solution of the problem, the analysis has been focused on the balanced cycle which is considered to be near optimal.

Two kinds of approximate solutions which also 
include the necessary conditions for realizing the balanced cycle has been derived. The accuracy of these analytical solutions has been confirmed by the comparisons with the numerical solution. These approximate solutions make it possible to know whether an operating condition gives a balanced cycle or not, to estimate the flow rate of air which is controlled automatically, and to determine the longitudinal distributions of brick temperature easily.

Simple expressions for the characteristics of hotblast stoves are obtained by the use of $\Phi-\Psi$ diagram. On the basis of these analysis, examples for the procedure of stove calculation are shown.

Furthermore, the approximate solution B proposed for the single blowing operation can be extended to the case of staggered parallel operation. ${ }^{18)}$

\section{Nomenclature}

A:

checkerwork heating surface area $\left(\mathrm{m}^{2}\right)$

$c_{a}, c_{g}, c_{s}:$

specific heats of air, of combustion product and of brick $\left(\mathrm{kcal} / \mathrm{kg} \cdot{ }^{\circ} \mathrm{C}\right)$

$D_{L}$ : domain of $\zeta$ where the longitudinal distribution of brick temperature is linear

$G=W_{g} c_{g} \Theta_{h} / W_{b} c_{a} \Theta_{c}=H_{h} \gamma / H_{c}$ : thermal capacity flow ratio referenced to blast volume $(-)$

$G^{*}=W_{g} c_{g} \Theta_{h} / \bar{W}_{a} c_{a} \Theta_{c}=G / \bar{u}$ : thermal capacity flow ratio referenced to the average flow rate of air (-)

$H_{c}=W_{b} c_{a} \Theta_{c} / M c_{s}, H_{h}=W_{g} c_{g} \Theta_{c} / M c_{s}: \quad(-)$

$h^{*}$ : $\quad$ overall heat transfer coefficient between gas and brick $\left(\mathrm{kcal} / \mathrm{m}^{2} \cdot \mathrm{hr} \cdot{ }^{\circ} \mathrm{C}\right)$

$L: \quad$ height of checkerwork $(\mathrm{m})$

$M: \quad$ mass of checkerwork $(\mathrm{kg})$

$\mathcal{N}$ : number of stoves

$T=T(\tau, \zeta)=\left(T^{\prime}-T_{a}^{\prime}\right) /\left(T_{g}^{\prime}-T_{a}^{\prime}\right):$ gas temperature $(-)$

$T_{b}=\left(T_{b}^{\prime}-T_{a}^{\prime}\right) /\left(T_{g}^{\prime}-T_{a}^{\prime}\right):$ dimensionless blast temperature $(-)$

$T^{\prime}: \quad$ gas temperature $\left({ }^{\circ} \mathrm{C}\right)$

$T_{a}^{\prime}: \quad$ temperature of cold air $\left({ }^{\circ} \mathrm{C}\right)$

$T_{b}^{\prime}: \quad$ blast temperature $\left({ }^{\circ} \mathrm{C}\right)$

$T_{g}^{\prime}$ : temperature of hot product by combustion $\left({ }^{\circ} \mathrm{C}\right)$

$t=t(\tau, \zeta)=\left(t^{\prime}-T_{a}^{\prime}\right) /\left(T_{g}^{\prime}-T_{a}^{\prime}\right)$ : brick temperature $(-)$

$t^{\prime}: \quad$ brick temperature $\left({ }^{\circ} \mathrm{C}\right)$

$t_{0}^{0}=t(0,0)$

$t_{1}^{1}=t(1,1)$

$U=1 /\left(1 / h_{c}^{*}+1 / h_{h}^{*} \gamma\right)(1+\gamma)$ overall heat transfer coefficient between combustion product and air $\left(\mathrm{kcal} / \mathrm{m}^{2} \cdot \mathrm{hr} \cdot{ }^{\circ} \mathrm{C}\right)$

$u=W_{a} / W_{b}$ : dimensionless flow rate of air (-)

$v: \quad$ parameter defined by Eq. (9) (-)

$W_{a}$ : mass flow rate of air through checkerwork $(\mathrm{kg} / \mathrm{hr})$

$W_{b}, W_{g}$ : mass flow rates of blast and of combustion product $(\mathrm{kg} / \mathrm{hr})$

$z$ : vertical coordinate from bottom of checkerwork $(\mathrm{m})$

$\alpha=1+\left(1 / \gamma h_{h}^{*} / h_{c}^{*}\right)(-)$

$\beta=\Lambda_{c} / \alpha T_{b} \quad(-)$

$\gamma=\Theta_{h} / \Theta_{c} \quad(-)$
$J t:$

amplitude of brick temperature oscillation in the middle region of checkerwork (-)

$\Delta \tau, \Delta_{\zeta}: \quad$ time interval and position interval $(-)$

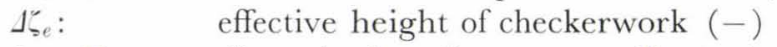

$\zeta=z / L: \quad$ dimensionless distance coordinate $(-)$

$\eta:$

$\Theta:$

$\theta$ :

$\Phi=1 /(1-\eta)(-)$

$\Psi=\Lambda_{c} / \alpha G(-)$

$A_{c}=h_{c}^{*} A / W_{b} c_{a}:$ modified Stanton number in cooling period (-)

$A_{h}=h_{h}^{*} A / W_{g} c_{g}:$ modified Stanton number in heating period (-)

$\mu_{y}=(f \mid y)(\partial y / \partial f):$ sensitivity coefficient $(-)$

$I I=H A=h^{*} A \Theta_{c} \mid M c_{s} \quad(-)$

$\tau=\theta / \Theta_{c}: \quad$ dimensionless time whose origin is placed

at the beginning of cooling period $(-)$

$\tau^{\prime}$ : dimensionless time whose origin is placed

Subscripts

$\mathrm{CP}, c$ : cooling period

$\mathrm{HP}, h$ : heating period

$m: \quad \int_{0}^{1} d \zeta$

$0: \quad \zeta=0$

1: $\quad \zeta=1$

num: numerical solution

appr: approximate solution

Superscripts

$\begin{array}{ll}-: & \int_{0}^{1} d \tau \text { or } \int_{0}^{\gamma} d \tau^{\prime} / \gamma \\ 0: & \tau=0 \\ 1: & \tau=1\end{array}$

\section{REFERENCES}

1) W. Nusselt: VDI-Zschft., 71 (1927), 85.

2) W. Nusselt: VDI-Zschft., 72 (1928), 1052.

3) H. Hausen: Z. angew. Math. Mech., 9 (1929), No. 3, 173.

4) H. Hausen: Z. angew. Math. Mech., 11 (1931), No. 2, 105.

5) G. Ackermann: Z. angew. Math. Mech., 11 (1931), 3, 192.

6) A. N. Nahavandi and A.S. Weinstein: Appl. Sci. Res., Section A, 10 (1961), 335.

7) J. M. Ridgion, A. J. Willmott, and J. H. Thewlis: Computer J., 7 (1964), 188.

8) A.J. Willmott: Int. J. Heat Mass Transfer, 7 (1964), 1291.

9) A. Schack: Z. f. techn. Physik, 9 (1928), No. 10, 390.

10) H. Hausen: Int. J. Heat Mass Transfer, 13 (1970), 1753.

11) V. Paschkis and R. Razelos: Iron Steel Eng., 42 (1966), No. 5, 115.

12) T. R. Schuerger and J. C. Agarwal: Iron Steel Eng., 37 (1961), 10, 143 .

13) S. L. Solomentsev: Stal in Eng., (1969), 538.

14) J. I. T. Green: JISI, 202 (1964), 833.

15) H. Hausen: VDI-Beiheft Verftk., (1942), No. 2, 31.

16) H. Kwakernaak, P. Tijssen and R. C. W. Strijbos: Automatica, 6 (1970), 33.

17) J. Schofield, P. Butterfield and P. A. Young: JISI, 199 (1961), 229

18) M. Horio, S. Niwa and I. Muchi: Tetsu-to-Hagané, 60 (1974), 622. 